\title{
Contraction Bidimensionality: the Accurate Picture $^{\star}$
}

\author{
Fedor V. Fomin ${ }^{1}$, Petr Golovach ${ }^{1}$, and Dimitrios M. Thilikos ${ }^{2}$ \\ 1 Department of Informatics, University of Bergen, N-5020 Bergen, Norway, \\ $\{$ fomin, Peter.Golovach\}@ii.uib.no \\ 2 Department of Mathematics, University of Athens, Panepistimioupolis, GR15784 \\ Athens, Greece, sedthilk@math.uoa.gr
}

\begin{abstract}
We provide new combinatorial theorems on the structure of graphs that are contained as contractions in graphs of large treewidth. As a consequence of our combinatorial results we unify and significantly simplify contraction bidimensionality theory - the meta algorithmic framework to design efficient parameterized and approximation algorithms for contraction closed parameters.
\end{abstract}

\section{Introduction}

The proof of Wagner's conjecture was the principal goal of the Graph Minors project of Robertson and Seymour. On the way to this goal, Robertson and Seymour have developed a powerful theory, which apparently became one of the most influential achievements in the modern Combinatorics. There are several very important consequences of Graph Minors theory for Algorithms Theory as well. In particular, obstruction theorems from Graph Minors give rise to powerful tools in the design of algorithms. Roughly speaking, such theorems say that either some width parameter (like pathwidth, treewidth, branchwidth, rank-width, etc.) of a graph is small (in which case one can proceed with dynamic programming techniques), or the graph contains some big pattern graph as a minor and such a graph can certify an answer to the problem. The most celebrated theorem of this type is the Excluding Grid-minor Theorem [15,13]: "there is a function $f$, such that every graph $G$ of treewidth at least $f(k)$ contains a $(k \times k)$-grid as a minor". Building on this theorem, Robertson and Seymour obtained their well known polynomial time algorithm for the disjoint path problems [14]. The bound in the obstruction theorem was refined by Robertson et al. [18] to $f(k)=20^{2 k^{5}}$. It is conjectured by Robertson et al. [18] that this bound is polynomial. For some classes of graphs like planar, graphs of bounded genus, and more generally, $H$-minor-free graphs, it is possible to prove that $f(k)=O(k)[4,5,18]$ and the bidimensionality theory was built on the top of these results. Bidimensionality

\footnotetext{
* The research of the first two authors was supported by the Norwegian Research Council. The research of the third author was Supported by the project "Kapodistrias" (АП 02839/28.07.2008) of the National and Kapodistrian University of Athens (project code: $70 / 4 / 8757$ ).
}

Dagstuhl Seminar Proceedings 09511

Parameterized complexity and approximation algorithms http://drops.dagstuhl.de/opus/volltexte/2010/2500 
provides a meta-algorithmic framework for the design of subexponential parameterized algorithms (i.e. the algorithms of running time $2^{o(k)} n^{O(1)}$, where $k$ is the parameter and $n$ the length of the input) for wide families of combinatorial problems. There is also an evidence that such subexponential algorithms are optimal even for sparse structures such as planar graphs (see surveys $[2,9]$ on bidimensionality). The applications of this theory are well understood and developed for minor closed graph optimization problems, i.e. the problems which only decrease under edge deletions/contractions.

However, many graph optimization problems are closed under edge contractions but not under edge deletions. Examples include most variants and extensions of the dominating set problem, connected dominating set, travelling salesman, or various distance modification problems. One of the challenges in Graph Theory and Algorithms is a possible extension of these results from graph minors to graph contractions (see $[2,7]$ ). The proofs from graph minors are very nontrivial, and such an extension is a difficult and sometimes an impossible task. For example, Demaine et al. in [7] disproved the analogue of Wagner' s Conjecture for graph contractions. Despite of that, it is possible to extend some algorithmic graph-minor results to graph contractions. In particular, it is possible to adapt obstruction theorems from graph minors to graph contractions. The extension of bidimensionality theory and meta theorems for contraction parameters was obtained in [3-5]. It is based on a modification of the grid-minor theorem for apex-minor-free graphs (graph class is apex-minor-free if it does not contain a graph with some fixed apex graph as a minor). An $(k \times k)$-augmented grid of span $s$ is an $(k \times k)$-grid with some extra edges such that each vertex is attached to at most $s$ non-boundary vertices of the grid. Fig. 1 provides an example of an augmented $(6 \times 6)$-grid of span 5 . The obstruction theorem for contractions $[3,5]$ states that for every apex graph $H$ there is a universal constant $c_{H}$ such that every $H$-minor-free graph of treewidth at least $c_{H} \cdot k$ can be contracted into a $(k \times k)$-augmented grid of span $c_{H}$. This combinatorial theorem is the cornerstone of the meta algorithmic framework in the design of subexponential parameterized algorithms for contraction-closed parameters on different classes of sparse graphs. This framework, called contraction bidimensionality, was developed by Demaine et al. [3] (see also surveys [2,9]). (We postpone the definitions related to contraction bidimensionality untill Section 5 , where we revise it.) Unfortunately, there is a drawback in the bidimensionality framework which is inherited by the "excluding-grid" theorem for contractions. The problem is that the number of augmented grids is huge. Even the number of planar augmented grids, i.e. graphs obtained by triangulating some faces of an $(k \times k)$-grid, is at least $2^{(k-1)^{2}}$. As a result, to verify if a parameter is apex-contraction bidimensional, one has to estimate its value on a graph family of exponential size. In this paper, we eliminate this main inefficiency of the meta algorithmic framework by redefining the notion of apex-contraction bidimensionality in simple and unifying way. With the new notion, we reduce the verification apex-contraction bidimensionality to the estimation of the value of the parameter in one specific triangulation of the $(k \times k)$-grid. The proof that this simple criterion holds for 
apex-contraction bidimensionality is highly nontrivial and requires the identification of a single pattern graph to which large-treewidth apex-minor free graphs can be contracted. This result has its own combinatorial merit as the contraction counterpart of the Excluding-grid Minor Theorem.

Let $\Gamma_{k}$ be the graph obtained from the $(k \times k)$-grid by triangulating internal faces of $(k \times k)$-grid such that all internal vertices become of degree 6 , all noncorner external vertices are of degree 4 , and then one corner of degree two is joined by edges with all vertices of the external face. Graph $\Gamma_{6}$ is shown in Fig. 1. The main combinatorial contribution of our work is the following theorem.
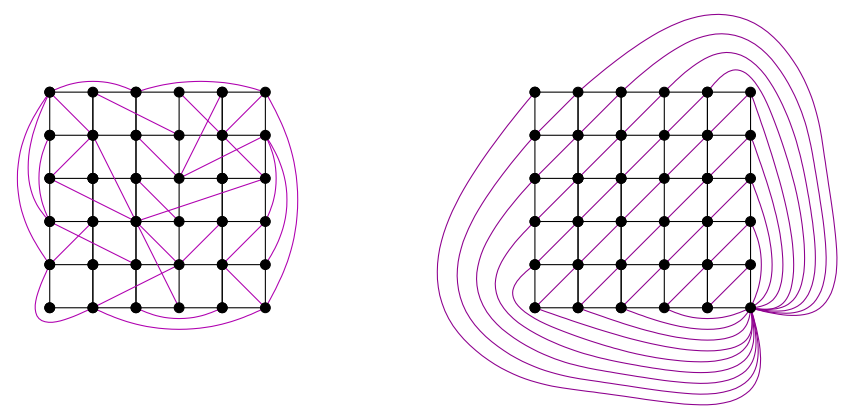

Fig. 1. An augmented $(6 \times 6)$-grid of span 5 (on the left) and the graph $\Gamma_{6}$ (on the right).

Theorem 1. Let $H$ be an apex graph. There is a constant $c_{H}$ such that every connected graph $G$ excluding $H$ as a minor and of treewidth at least $c_{H} \cdot k$, contains $\Gamma_{k}$ as a contraction.

In the conclusion of their survey [2], Demaine and Hajiaghayi mentioned that contraction bidimensionality is so far undefined for $H$-minor-free graphs (or general graphs). They also wrote that it would be quite interesting to explore an analogous theory of graph contractions paralleling the Graph Minor Theory. In this direction, we make a step by extending Theorem 1 for more general graph classes. We prove that for $H$-minor free graphs, big treewidth implies the existence of one two pattern graphs as contractions. In what follows, $\operatorname{tw}(G)$ denotes the treewidth of $G$.

Theorem 2. Let $G$ be a connected graph excluding a graph $H$ as a minor. Then there exists some constant $c_{H}$ such that if $\operatorname{tw}(G) \geq c_{H} \cdot k^{2}$, then $G$ contains as a contraction either $\Gamma_{k}$ or $\Pi_{k}$, where $\Pi_{k}$ is the graph obtained from $\Gamma_{k}$ by adding a vertex adjacent to all vertices of $\Gamma_{k}$.

We stress that one can (artificially) define contraction-closed parameters whose value is non-trivial for $\Pi_{k}$ and, for them, bidimensionality could be defined even for $\mathrm{H}$-minor free graphs. However, it is interesting to notice that for 
all known contraction-closed parameters the value of $\Pi_{k}$ is independent from $k$ and this indicates that the classic Win/win approach for contraction closed parameters is structurally confined to apex-minor free graphs.

Finally, we show the following analogue of the Excluding Grid-minor Theorem. It follows that, instead of a single grid, there are three pattern graphs appearing as contractions in graphs with big treewidth (for minors, the only such pattern is the grid).

Theorem 3. For any positive integer $k$, there is a constant $c_{k}$ such that every connected graph $G$ where $\operatorname{tw}(G) \geq c_{k}$, contains one of $K_{k}, \Gamma_{k}, \Pi_{k}$ as a contraction, where $K_{k}$ is a complete graph on $k$ vertices.

\section{Basic definitions}

We consider finite undirected graphs without loops or multiple edges. The vertex set of a graph $G$ is denoted by $V(G)$ and its edge set by $E(G)$.

Let $G$ be a graph. For a vertex $v$, we denote by $N_{G}(v)$ its (open) neighborhood, i.e. the set of vertices which are adjacent to $v$. The closed neighborhood of $v$, i.e. the set $N_{G}(v) \cup\{v\}$, is denoted by $N_{G}[v]$. For $U \subseteq V(G)$, we define $N_{G}[U]=$ $\bigcup_{v \in U} N_{G}[v]$ (we may omit index if the graph under consideration is clear from the context). If $U \subseteq V(G)$ (resp. $u \in V(G)$ or $E \subset E(G)$ or $e \in E(G)$ ) then $G-U$ (resp. $G-u$ or $G-E$ or $G-e$ ) is the graph obtained from $G$ by the removal of vertices of $U$ (resp. of vertex $u$ or edges of $E$ or of the edge $e$ ).

Surfaces. A surface $\Sigma$ is a compact 2-manifold without boundary (we always consider connected surfaces). Whenever we refer to a $\Sigma$-embedded graph $G$ we consider a 2-cell embedding of $G$ in $\Sigma$. To simplify notations, we do not distinguish between a vertex of $G$ and the point of $\Sigma$ used in the drawing to represent the vertex or between an edge and the line representing it. We also consider a graph $G$ embedded in $\Sigma$ as the union of the points corresponding to its vertices and edges. That way, a subgraph $H$ of $G$ can be seen as a graph $H$, where $H \subseteq G$. Recall that $\Delta \subseteq \Sigma$ is an open (resp. closed) disc if it is homeomorphic to $\left\{(x, y): x^{2}+y^{2}<1\right\}$ (resp. $\left.\left\{(x, y): x^{2}+y^{2} \leq 1\right\}\right)$. The Euler genus of a nonorientable surface $\Sigma$ is equal to the non-orientable genus $\tilde{g}(\Sigma)$ (or the crosscap number). The Euler genus of an orientable surface $\Sigma$ is $2 g(\Sigma)$, where $g(\Sigma)$ is the orientable genus of $\Sigma$. We refer to the book of Mohar and Thomassen [12] for more details on graphs embeddings.

Contractions and minors. Given an edge $e=\{x, y\}$ of a graph $G$, the graph $G / e$ is obtained from $G$ by contracting the edge $e$, i.e. the endpoints $x$ and $y$ are replaced by a new vertex $v_{x y}$ which is adjacent to the old neighbors of $x$ and $y$ (except from $x$ and $y$ ). A graph $H$ obtained by a sequence of edge-contractions is said to be a contraction of $G$. In this work we use contraction with different topological properties, and for this purpose it is convenient to give an alternative definition of contraction. 
Let $G$ and $H$ be graphs and let $\phi: V(G) \rightarrow V(H)$ be a surjective mapping such that

1. for every vertex $v \in V(H)$, its codomain $\phi^{-1}(v)$ induces connected graph $G\left[\phi^{-1}(v)\right]$

2. for every edge $\{v, u\} \in E(H)$, the graph $G\left[\phi^{-1}(v) \cup \phi^{-1}(u)\right]$ is connected;

3. for every $\{v, u\} \in E(G)$, either $\phi(v)=\phi(u)$, or $\{\phi(v), \phi(u)\} \in E(H)$.

We say that $H$ is a contraction of $G$ via $\phi$, and denote it as $H \leq_{c}^{\phi} G$. Let us observe that $H$ is a contraction of $G$ if $H \leq_{c}^{\phi} G$ for some $\phi: V(G) \rightarrow V(H)$. In this case we simply write $H \leq_{c} G$. If $H \leq_{c}^{\phi} G$ and $v \in V(H)$ then we call the codomain $\phi^{-1}(v)$ by the model of $v$ in $G$.

Let $G$ be a graph embedded in some surface $\Sigma$ and let $H$ be a contraction of $G$ via function $\phi$. We say that $H$ is a surface contraction of $G$ if for each vertex $v \in V(H), G\left[\phi^{-1}(v)\right]$ is embedded in some open disk in $\Sigma$.

Let $G_{0}$ be a graph embedded in some surface $\Sigma$ of Euler genus $\gamma$ and let $G^{+}$be another graph that might share common vertices with $G_{0}$. We set $G=G_{0} \cup G^{+}$. Let also $H$ be some graph and let $v \in V(H)$. We say that $G$ contains a graph $H$ as a v-smooth contraction if $H \leq_{c}^{\phi} G$ for some $\phi: V(G) \rightarrow V(H)$ and there exists an closed disk $D$ in $\Sigma$ such that all the vertices of $G$ that are outside $D$ are exactly the model of $v$, i.e. $\phi^{-1}(v)=V(G) \backslash(V(G) \cap D)$.

A graph $H$ is a minor of a graph $G$ if $H$ is the contraction of some subgraph of $G$ and we denote it $H \leq_{m} G$. It is said that $H$ is a surface minor of a graph $G$ embedded in some surface $\Sigma$ if $H$ is the surface contraction of some subgraph of $G$. It can be easily noted that if $H$ is a surface minor of a graph $G$ embedded in a surface $\Sigma$ then it can be assumed that $H$ is embedded in a surface $\Sigma^{\prime}$ homeomorphic to $\Sigma$. For simplicity, we assume in such cases that $\Sigma^{\prime}$ and $\Sigma$ are the same surface.

We say that a graph $G$ is $H$-minor-free when it does not contain $H$ as a minor. We also say that a graph class $\mathcal{G}$ is $H$-minor-free (or, excludes $H$ as a minor) when all its members are $H$-minor-free. An apex graph is a graph obtained from a planar graph $G$ by adding a vertex and making it adjacent to some of the vertices of $G$. A graph class $\mathcal{G}$ is apex-minor-free if $\mathcal{G}$ excludes a fixed apex graph $H$ as a minor.

Grids and their triangulations. Let $k$ and $r$ be positive integers where $k, r \geq$ 2. The $(k \times r)$-grid is the Cartesian product of two paths of lengths $k-1$ and $r-1$ respectively. A vertex of a $(k \times r)$-grid is a corner if it has degree 2 . Thus each $(k \times r)$-grid has 4 corners. A vertex of a $(k \times r)$-grid is called internal if it has degree 4 , otherwise it is called external.

A partial triangulation of a $(k \times r)$-grid is a planar graph obtained from a $(k \times r)$-grid (we call it the underlying grid) by adding edges. Let us note that there are many non-isomorphic partial triangulations of on underlying grid. For each partial triangulation of a $(k \times r)$-grid we use the terms corner, internal and external referring to the corners, the internal and the external vertices of the underlying grid.

Let us remind that we define $\Gamma_{k}$ as the following (unique, up to isomorphism) triangulation of a plane embedding of the $(k \times k)$-grid. Let $\Gamma$ be a plane em- 
bedding of the $(k \times k)$-grid such that all external vertices are on the boundary of the external face. We triangulate internal faces of the $(k \times k)$-grid such that all the internal vertices have degree 6 in the obtained graph and all non-corner external vertices have degree 4 , and then one corner of degree two is joined by edges with all vertices of the external face (we call this corner loaded). We also use notation $\Gamma_{k}^{*}$ for the graph obtained from $\Gamma_{k}$ if we remove all edges incident to its loaded vertex that do not exist in its underlying grid. We define the graph $\Pi_{k}$ as the graph obtained if we add a new vertex in $\Gamma_{k}$ and connect it with all vertices of it. Let $K$ be a clique of size 3 in $\Gamma_{k}^{*}$. Notice that exactly two of the edges of $\Gamma_{k}[K]$ are also edges of the underlying $(k \times k)$-grid of $\Gamma_{k}$. We call the unique vertex of $K$ that is incident to both these two edges rectangular vertex of $K$.

Let $G$ be a partial triangulation of a $(k \times k)$-grid and let $m$ be a positive integer. Then by $\mathbf{P}_{m}(G)$ we denote the collection of $m^{2}$ vertex disjoint induced subgraphs of $G$ where all of them are isomorphic to a $(\lfloor k / m\rfloor \times\lfloor k / m\rfloor)$-grid and where the union of their vertices induce a graph containing $(\lfloor k / m\rfloor \cdot m \times$ $\lfloor k / m\rfloor \cdot m)$-grid as a spanning subgraph.

Suppose that $G$ is a connected graph which contains as an induced subgraph a partially triangulated $((k+2) \times(k+2))$-grid $\Gamma$ in such a way that internal vertices of $\Gamma$ are not adjacent to vertices of $V(G) \backslash V(\Gamma)$. We define the boundary contraction of $G$ to $\Gamma$ as the partially triangulated $(k \times k)$-grid $\mathbf{b c}(G, \Gamma)$ obtained as follows: let $v$ be a corner of the subgrid of $\Gamma$ induced by the internal vertices which has the minimum degree (in this graph), all external vertices of $\Gamma$ are contracted to $v$, and then all vertices of $V(G) \backslash V(\Gamma)$ are contracted to $v$. Note that if $\Gamma$ is embedded in a disk of some surface $\Sigma$ then $\mathbf{b c}(G, \Gamma)$ is a $v$-smooth contraction of $G$.

Treewidth and pathwidth. A tree decomposition of a graph $G$ is a pair $(\mathcal{X}, T)$ where $T$ is a tree and $\mathcal{X}=\left\{X_{i} \mid i \in V(T)\right\}$ is a collection of subsets of $V(G)$ such that:

1. $\bigcup_{i \in V(T)} X_{i}=V(G)$,

2. for each edge $\{x, y\} \in E(G),\{x, y\} \subseteq X_{i}$ for some $i \in V(T)$; and

3. for each $x \in V(G)$ the set $\left\{i \mid x \in X_{i}\right\}$ induces a connected subtree of $T$.

The width of a tree decomposition $\left(\left\{X_{i} \mid i \in V(T)\right\}, T\right)$ is $\max _{i \in V(T)}\left\{\left|X_{i}\right|-\right.$ $1\}$. The treewidth of a graph $G$ is the minimum width over all tree decompositions of $G$. If, in the above definitions, we restrict the tree $T$ to be a path then we define the notions of path decomposition and pathwidth. We write $\operatorname{tw}(G)$ and pw $(G)$, respectively, for the treewidth and the pathwidth of a graph $G$.

Graph minor theorem. The proof of our results is using the Excluded Minor Theorem from the Graph Minor theory. Before we state it, we need some definitions.

Definition 1 (Clique-Sums). Let $G_{1}$ and $G_{2}$ be two disjoint graphs, and $k \geq 0$ an integer. For $i=1,2$, let $W_{i} \subseteq V\left(G_{i}\right)$, form a clique of size $h$ and let $G_{i}^{\prime}$ be the graph obtained from $G_{i}$ by removing a set of edges (possibly empty) from the 
clique $G_{i}\left[W_{i}\right]$. Let $F: W_{1} \rightarrow W_{2}$ be a bijection between $W_{1}$ and $W_{2}$. We define the $h$-clique-sum of $G_{1}$ and $G_{2}$, denoted by $G_{1} \oplus_{h, F} G_{2}$, or simply $G_{1} \oplus G_{2}$ if there is no confusion, as the graph obtained by taking the union of $G_{1}^{\prime}$ and $G_{2}^{\prime}$ by identifying $w \in W_{1}$ with $F(w) \in W_{2}$, and by removing all the multiple edges. The image of the vertices of $W_{1}$ and $W_{2}$ in $G_{1} \oplus G_{2}$ is called the join of the sum.

Note that some edges of $G_{1}$ and $G_{2}$ are not edges of $G$, since it is possible that they had edges which were removed by clique-sum operation. Such edges are called virtual edges of $G$. We remark that $\oplus$ is not well defined; different choices of $G_{i}^{\prime}$ and the bijection $F$ could give different clique-sums. A sequence of $h$-clique-sums, not necessarily unique, which result in a graph $G$, is called a clique-sum decomposition of $G$.

Definition 2 (h-nearly embeddable graphs). Let $\Sigma$ be a surface with cycles $C_{1}$, $\ldots, C_{h}$, such that each cycle $C_{i}$ is the border of an open disc $\Delta_{i}$ in $\Sigma$. A graph $G$ is h-nearly embeddable in $\Sigma$, if $G$ has a subset $X$ of size at most $h$, called apices, so that there are (possibly empty) subgraphs $G_{0}, \ldots, G_{h}$ of $G \backslash X$ where

i) $G^{\prime}=G \backslash X=G_{0} \cup G_{1} \cup \cdots \cup G_{h}$ (we denote $G^{+}=G_{1} \cup \ldots \cup G_{h}$ ),

ii) $G_{0}$ is embeddable in $\Sigma$ such that $G_{0} \cap \bigcup_{i=1, \ldots, h} \Delta_{h}=\emptyset$, we fix an embedding of $G_{0}$,

iii) graphs $G_{1}, \ldots, G_{h}$ (called vortices) are pairwise disjoint,

iv) for $1 \leq i \leq h$, let $U_{i}:=\left\{u_{1}^{i}, \ldots, u_{m_{i}}^{i}\right\}=V\left(G_{0}\right) \cap V\left(G_{i}\right)$, $G_{i}$ has a path decomposition $\mathcal{B}_{i}=\left(B_{j}^{i}\right)_{1 \leq j \leq m_{i}}$, of width at most $h$ such that

a) for $1 \leq j \leq m_{i}$ we have $u_{j}^{i} \in B_{j}^{i}$,

b) the vertices (we call them bases of $G_{i}$ ) $u_{1}^{i}, \ldots, u_{m_{i}}^{i}$ appear on $C_{i}$ in this order (either if we walk clockwise or anti-clockwise).

The following proposition is known as the Excluded Minor Theorem [17] and is the cornerstone of Robertson and Seymour's Graph Minors theory.

Theorem 4 ([17]). For every non-planar graph $H$, there exists an integer $c_{H}$, depending only on $H$, such that every graph excluding $H$ as a minor can be obtained by $c_{H}$-clique-sums from graphs that can be $c_{H}$-nearly embedded in a surface $\Sigma$ in which $H$ cannot be embedded. Moreover, while applying each of the clique sums, at most three vertices from each summand other than apices and vertices in vortices are identified.

Let us remark that by the result of Demaine et al. [6] such a clique-sum decomposition can be obtained in time $O\left(n^{c}\right)$ for some constant $c$ which depends only from $H$ (see also [1]).

Lemmata on treewidth. We need the following two well known results about treewidth.

Lemma 1. If $G_{1}$ and $G_{2}$ are graphs, then $\mathbf{t w}\left(G_{1} \oplus G_{2}\right) \leq \max \left\{\mathbf{t w}\left(G_{1}\right), \operatorname{tw}\left(G_{2}\right)\right\}$.

Lemma 2. If $G$ is a graph and $X \subseteq V(G)$, then $\mathbf{t w}(G-X) \geq \operatorname{tw}(G)-|X|$.

The following lemma is implicit in the proofs from $[5,4]$.

Lemma 3. Let $G$ be a h-nearly embeddable graph without apices (i.e. where $X=\emptyset)$. Then $\mathbf{t w}(G) \leq(h+1) \cdot\left(\mathbf{t w}\left(G_{0}\right)+1\right)-1$. 


\section{Lemmata on grids and their triangulations}

In this section we give a sequence of auxiliary lemmata used to prove Lemma 11, the most important technical tool in the proofs of Theorems 1 and 2 .

It is implicit in the proofs in [4, Theorem 4.12] and [16, (5.1)] that if the treewidth of a graph embedded in a surface with Euler genus eg $(G)$ is large enough then this graph contains $(r \times r)$-grid as a surface minor. We state this with the following lemmata.

Lemma 4. Let $G$ be a graph embedded in a surface $\Sigma$ of Euler genus $\gamma$. If the treewidth of $G$ is more than $12 r(\gamma+1)$, then $G$ has the $(r \times r)$-grid as a surface minor.

Lemma 5. Let $H$ be a partial triangulation of a $((2 k+1) \times(2 k+1))$-grid. Then $H$ contains $\Gamma_{k}$ as a contraction in a way that all external vertices of $H$ belong to the model of the loaded corner of $\Gamma_{k}$.

A basic ingredient of our proofs is a result roughly stating that if a graph $G$ with a big grid as a minor is embedded on a surface $\Sigma$ of small genus, then there is a disc in $\Sigma$ containing a big enough part of the grid of $G$. This result is implicit in the work of Robertson and Seymour and there are simpler alternative proofs by Mohar and Thomassen [11, 19] (see also [4, Lemma 3.3] and [8, Lemma 4.7]). By using a variant of this result from Geelen et al. [10] together with Lemmata 4 and 5 we prove the following.

Lemma 6. Let $G$ be a graph embedded in a surface of Euler genus $\gamma$ and let $k$ be a positive integer. If the treewidth of $G$ is more than $12 \cdot(\gamma+1)^{3 / 2} \cdot(2 k+4)$, then $G$ contains $\Gamma_{k}$ as a v-smooth contraction with $v$ being one of the corners of $\Gamma_{k}$.

The following is based on Lemma 3 and Lemma 6

Lemma 7. There is a constant $c$ such that if $G$ is a graph h-nearly embedded in a surface of Euler genus $\gamma$ without apices, where $\operatorname{tw}(G) \geq c \cdot \gamma^{3 / 2} \cdot h^{3 / 2} \cdot k$, then $G$ contains as a v-smooth contraction the graph $\Gamma_{k}$ with the loaded corner $v$.

Let $\mathcal{C}=\left\{K_{1}, \ldots, K_{r}\right\}$ be a sequence of (not necessary different) cliques in a graph $G$ and let $E \subseteq E\left(G\left[\cup_{i=1, \ldots, r} K_{i}\right]\right)$. We define the $\operatorname{cl}(G, \mathcal{C}, E)$ to be the graph constructed from $G-E$ by adding for each non-empty $K_{i}$ a new vertex $z_{\text {new }}^{(i)}$ and making it adjacent to all vertices in $K_{i}$.

The proof of the following lemma is based on Lemma 5.

Lemma 8. Let $G_{0}$ be a graph embedded in surface $\Sigma$ of Euler genus $\gamma$ and let $G^{+}$be another graph that might share common vertices with $G_{0}$. We set $G^{\prime}=G_{0} \cup G^{+}$. Let $\mathcal{C}=\left\{K_{1}, \ldots, K_{r}\right\}$ be a collection of cliques in $G^{\prime}$ such that each of them shares at most 3 vertices with $G_{0}$. Let $E \subseteq E\left(G^{\prime}\left[\cup_{i=1, \ldots, r} K_{i}\right]\right)$ and let $\hat{G}^{\prime}=\operatorname{cl}\left(G^{\prime}, \mathcal{C}, E\right)$. Then, if $G^{\prime}$ contains $\Gamma_{2 k+5}$ with the loaded corner $v$ as a $v$-smooth contraction, then $\hat{G}^{\prime}$ contains $\Gamma_{k}$ as a contraction. 
We also need the following two lemmata.

Lemma 9. Let $G$ be a graph and let $\mathcal{C}=\left\{K_{1}, \ldots, K_{r}\right\}$ be a sequence of cliques in $G$, let $E \subseteq E\left(G\left[\cup_{i=1, \ldots, r} K_{i}\right]\right)$ and let $\hat{G}=\operatorname{cl}(G, \mathcal{C}, E)$. Let also $G^{\prime}=G-X$ for some $X \subseteq V(G)$, where $|X| \leq h$. We set $\mathcal{C}^{\prime}=\left\{K_{1} \backslash X, \ldots, K_{r} \backslash X\right\}$, E' be the edges of $E$ without endpoints in $X$ and let $\hat{G}^{\prime}=\operatorname{cl}\left(G^{\prime}, \mathcal{C}^{\prime}, E^{\prime}\right)$. Then if $\hat{G}^{\prime}$ can be contracted to $\Gamma_{k}$, then $\hat{G}$ can be contracted to a graph $H$ containing a vertex subset $Y,|Y| \leq h$, where $H-Y=\Gamma_{k}$.

Lemma 10. Let $G$ be a connected graph that obtained from $\Gamma_{2^{r} k+4\left(2^{r}-1\right)}$ by adding $r \geq 1$ new vertices and an arbitrary number of edges incident to these vertices. Then $G$ can be contracted to an apex graph which contains $\Gamma_{k}$ and at most one additional vertex which is adjacent to some vertices of $\Gamma_{k}$.

The following lemma is the most crucial technical result used in the proofs of Theorems 1 and 2.

Lemma 11. Let $G$ be a connected graph excluding a graph $H$ as a minor. Then there exists some constant $c_{H}$ such that if $\operatorname{tw}(G) \geq c_{H} \cdot k$, then $G$ contains as a contraction a graph where the removal of at most one of its vertices results to $\Gamma_{k}$.

Proof. Let $G$ be a connected $H$-minor-free graph. If $H$ is a planar graph then $G$ has bounded treewidth [13] and the claim of the theorem is trivial. Assume that $H$ is not planar. By Theorem $4, G$ can be represented as $h$-clique-sum $G=G_{1} \oplus \cdots \oplus G_{m}$ such that each graph $G_{i}$ can be $h$-nearly-embedded in a surface $\Sigma$ (on which $H$ cannot be embedded) where $h$ is a constant which depends only on $H$. Let $F=G_{i}$ such that $\operatorname{tw}(F)=\max _{j=1, \ldots, m} \operatorname{tw}\left(G_{j}\right)$. By Lemma 1,

$$
\operatorname{tw}(G) \leq \mathbf{t w}(F) .
$$

Assume that $F$ is $h$-nearly-embedded in $\Sigma$ and denote by $X$ the set of apices of $F$. Recall that $|X| \leq h$. Let $F^{\prime}=F-X$. By Lemma 2,

$$
\operatorname{tw}(F)-|X| \leq \mathbf{t w}\left(F^{\prime}\right) .
$$

Observe that $F^{\prime}$ is $h$-nearly embedded in $\Sigma$ without apices. Using Lemma 7 and combining it's claim with inequalities 1 and 2 , we note that there is a constant $c_{H}$ which depends only on $H$ such that if $\operatorname{tw}(G) \geq c_{H} \cdot k$ then $F^{\prime}$ contains as a $v$-smooth contraction the graph $\Gamma_{r}$ where $v$ is the loaded corner of $\Gamma_{r}$ and $r=2^{r+1} \cdot k+8\left(2^{r}-1\right)+5$.

Denote by $S_{1}, \ldots, S_{t}$ components of the graph $G-V(F)$. For each $S_{i}$ let $K_{i}$ be the set of vertices of $F$ which are adjacent to some vertex of $S_{i}$, and let $\mathcal{C}=\left\{K_{1}, \ldots, K_{t}\right\}$. By the definition of $h$-clique-sum each $K_{i}$ is a clique of $F$. Denote by $E$ the set of virtual edges of $F$. We assume that for any virtual edge $\{u, v\}$, there is a clique $K_{i} \in \mathcal{C}$ such that $u, v \in K_{i}$ (otherwise it is easy to redefine $h$-clique-sums in the representation of $G$ and exclude such an edge). For every component $S_{i}$, all vertices of it are contracted into single vertex $z_{\text {new }}^{(i)}$. 
Denote by $\hat{F}$ obtained from $G$ by these contractions. It can be easily seen that $\hat{F}$ is the graph $\operatorname{cl}(F, \mathcal{C}, E)$. We set $\mathcal{C}^{\prime}=\left\{K_{1} \backslash X, \ldots, K_{t} \backslash X\right\}, E^{\prime}$ be the edges of $E$ without endpoints in $X$ and let $\hat{F}^{\prime}=\operatorname{cl}\left(F^{\prime}, \mathcal{C}^{\prime}, E^{\prime}\right)$. Since $F^{\prime}$ can be contracted to $\Gamma_{r}$, it follows immediately from Lemma 8 that $\hat{F}^{\prime}$ contains $\Gamma_{s}$ as a contraction for $s=(r-6) / 2=2^{r} \cdot k+4\left(2^{r}-1\right)$. Then by Lemma $9, \hat{F}$ (and consequently the graph $G$ ) can be contracted to a graph $R$ containing a vertex subset $Y,|Y| \leq h$ such that $R-Y=\Gamma_{s}$. It remains to use Lemma 10 and note that $R$ can be contracted to an apex graph which consists of $\Gamma_{k}$ and at most one apex vertex which is adjacent to some vertices of $\Gamma_{k}$. The graph $R$ is a contraction of $G$, so $G$ contains as a contraction a graph which after the removal of at most one of its vertices results to $\Gamma_{r}$.

\section{Proofs of theorems}

Proof (of Theorem 1). Let $H$ be an apex graph. It was shown by Robertson et al. [13], that every planar graph on $\lceil h / 7\rceil$ vertices is a minor of an $(h \times h)$-grid, and without loss of generality, we can assume that $H$ is a graph constructed from a $(h \times h)$-grid by adding one apex vertex adjacent to all vertices of the grid. By Lemma 11, if $\operatorname{tw}(G) \geq c_{H} \cdot k$, for some constant $c_{H}$, then $G$ contains as a contraction a graph $F$ such that the removal of at most one of its vertices results in $\Gamma=\Gamma_{h \cdot(k+2)}$. If $F=\Gamma$, then the theorem follows trivially. Thus we assume that $F$ has an additional vertex $u$ adjacent to some vertices of $\Gamma$. We consider the collection $\mathbf{P}_{h}(\Gamma)$ of $h^{2}$ vertex disjoint induced subgraphs of $\Gamma$. We claim that there is a subgraph in $\mathbf{P}_{h}(\Gamma)$ such that none of its vertices is adjacent to $u$. Indeed, if each subgraph in $\mathbf{P}_{h}(\Gamma)$ contains a vertex adjacent to $u$, then $\Gamma$ contain an $(h \times h)$-grid as a minor such that the nodes of this grid are the neighbors of $u$. But this contradicts the assumption that $G$ is $H$-minor-free.

Thus there is a subgraph $\Gamma_{k+2}^{*}$ in $\mathbf{P}_{h}(\Gamma)$ such that none of the vertices of $\Gamma_{k+2}^{*}$ is adjacent to $u$. The graph $\Gamma_{k+2}^{*}$ can be seen as a graph obtained from $\Gamma_{k+2}$ by removal all edges adjacent to the loaded corner of $\Gamma_{k+2}$ that are not the edges of the underlying grid. Therefore, after applying the boundary contraction of $F$ to $\Gamma_{k+2}^{*}$, the resulting $\operatorname{graph} \mathbf{b c}\left(F, \Gamma^{\prime}\right)$ is $\Gamma_{k}$.

Proof (of Theorem 2). Let us assume that $\operatorname{tw}(G) \geq c_{H} \cdot k^{2}$, where $c_{H}$ is the constant from Lemma 11. By the same lemma, $G$ can be contracted to a graph $H$ such that by the removal of at most one vertex of $H$ the result is isomorphic to $\Gamma_{k^{2}}$. If $H$ is itself isomorphic to $\Gamma_{k^{2}}$ then we are done as $\Gamma_{k^{2}}$ contains $\Gamma_{k}$ as a contraction. Suppose then that $G$ has an additional vertex $x$ and let $S=N_{G}(x)$. Let $\mathcal{P}$ be a collection of $k$ disjoint copies of $\Gamma_{k}^{*}$ in $\Gamma_{k^{2}}$. In case for some $A \in \mathcal{P}$, $V(A) \cap S=\emptyset$, we contract all edges with both endpoints in $\cup_{H \in \mathcal{P} \backslash\{A\}} V(H)$. The obtained graph is $\Gamma_{k}^{*}$ with one more vertex adjacent to all its external vertices and this graph can be further contracted to $\Gamma_{k}$. Suppose now that each graph in $\mathcal{P}$ intersects some neighbor of $x$. Then contract all edges of all graphs in $\mathcal{P}$ and the resulting graph is $P_{k}$.

Proof (of Theorem 3). Suppose that $G$ does not contain $H=K_{k}$ as a contraction. Then $G$ is an $H$-minor-free graph. By Theorem 2, there exists some 
constant $c_{H}$ such that if $\operatorname{tw}(G) \geq c_{H} \cdot k^{2}$, then $G$ contains as a contraction either $\Gamma_{k}$ or $\Pi_{k}$. We put $c_{k}=c_{H} \cdot k^{2}$, which concludes the proof of the theorem.

\section{Contraction bidimensionality revised}

The theory of bidimensionality is a meta algorithmic framework for designing efficient fixed-parameter algorithms and approximation algorithms for a broad range of graph problems. Roughly speaking, graph problem is bidimensional if (a) the solution of the problem on the $(k \times k)$-grid (or some modification of the grid) is proportional to $\Omega\left(k^{2}\right)$ (b) the problem is closed under taking minor/contraction, which means that the solution value can only decrease with contracting or removing edges in the graph. Many problems are bidimensional. Classic examples are vertex cover, dominating set, and feedback vertex set.

A parameter $P$ is a function mapping graphs to nonnegative integers. The decision problem associated with $P$ asks, for a given graph $G$ and nonnegative integer $k$, whether $P(G) \leq k$. Intuitively, a parameter is bidimensional if its value depends on the area of a grid and not on its width or height.

For minor-closed parameters, the definition of bidimensionality is easy [3]. A parameter $P$ is minor bidimensional if (a) $P$ is closed under taking of minors and (b) for the $(k \times k)$-grid $\Gamma, P(\Gamma)=\Omega\left(k^{2}\right)$. Examples of minor bidimensional parameters are sizes of a vertex cover, a feedback vertex set, or a minimum maximal matching in a graph.

For contraction-closed parameters, the definition of bidimensionality is much more complicated and depends on the class of graphs it is used for. Demaine et al. $[3,4,2,8]$ defined parameter $P$ as contraction bidimensional if the following hold: (a) $P$ is closed under taking of contractions and (b) for a " $(k \times k)$-gridlike graph" $\Gamma, P(\Gamma)=\Omega\left(k^{2}\right)$. Here the property of being "grid-like graph" is different for different graph classes and is defined as follows.

b1) For planar graphs and single-crossing-minor-free graphs, a " $(k \times k)$-grid-like graph" is a partially triangulated $(k \times k)$-grid;

b2) For graphs of Euler genus $\gamma$, this is a partially triangulated $(k \times k)$-grid with up to $\gamma$ additional handles;

b3) For apex-minor-free graphs, this is $(k \times k)$-augmented grid, i.e. partially triangulated grid augmented with additional edges such that each vertex is incident to $O(1)$ edges to non-boundary vertices of the grid.

Typical examples of contraction bidimensional parameters are sizes of a dominating, clique-transversal, or edge domination sets.

The main contribution of Theorem 1 to contraction bidimensionality is that the notions of "grid-like" graphs (b1), (b2), and (b3) can be replaced by the following one

$\left.\mathbf{b}^{\prime}\right) P\left(\Gamma_{k}\right)=\Omega\left(k^{2}\right)$.

This is justified by the following theorem, which is the main (meta) algorithmic contribution of this paper. 
Theorem 5. Let $P$ be a graph parameter which satisfies conditions (a) and $\left(\mathbf{b}^{\prime}\right)$. Let $G$ be a n-vertex graph excluding an apex graph $H$ as a minor. Then if $P$ is computable in time $2^{O(\mathbf{t w}(G))} \cdot n^{O(1)}$, then deciding $P(G) \leq k$ can be done in time $2^{O(\sqrt{k})} \cdot n^{O(1)}$.

\section{References}

1. A. Dawar, M. Grohe, And S. Kreutzer, Locally excluding a minor, in LICS'07, IEEE Computer Society, 2007, pp. 270-279.

2. E. Demaine And M. Hajiaghayi, The bidimensionality theory and its algorithmic applications, The Computer Journal, 51 (2007), pp. 292-302.

3. E. D. Demaine, F. V. Fomin, M. Hajiaghayi, and D. M. Thilikos, Bidimensional parameters and local treewidth, SIAM J. Discrete Math., 18 (2004/05), pp. 501-511.

4. - Subexponential parameterized algorithms on graphs of bounded genus and H-minor-free graphs, J. ACM, 52 (2005), pp. 866-893.

5. E. D. Demaine And M. Hajiaghayi, Linearity of grid minors in treewidth with applications through bidimensionality, Combinatorica, 28 (2008), pp. 19-36.

6. E. D. Demaine, M. Hajiaghayi, and K. IChi Kawarabayashi, Algorithmic graph minor theory: Decomposition, approximation, and coloring, in FOCS'05, IEEE Computer Society, 2005, pp. 637-646.

7. — Algorithmic graph minor theory: Improved grid minor bounds and Wagner's contraction, Algorithmica, (2009), p. to appear.

8. E. D. Demaine, M. Hajiaghayi, and D. M. Thilikos, The bidimensional theory of bounded-genus graphs, SIAM J. Discrete Math., 20 (2006), pp. 357-371 (electronic).

9. F. Dorn, F. V. Fomin, And D. M. Thilikos, Subexponential parameterized algorithms, Comp. Sci. Rev., 2 (2008), pp. 29-39.

10. J. F. Geelen, R. B. Richter, And G. Salazar, Embedding grids in surfaces, European J. Combin., 25 (2004), pp. 785-792.

11. B. Mohar, Combinatorial local planarity and the width of graph embeddings, Canad. J. Math., 44 (1992), pp. 1272-1288.

12. B. Mohar and C. Thomassen, Graphs on surfaces, Johns Hopkins University Press, Baltimore, MD, 2001.

13. N. Robertson, P. Seymour, And R. Thomas, Quickly excluding a planar graph, J. Combin. Theory Ser. B, 62 (1994), pp. 323-348.

14. N. Robertson And P. D. Seymour, Disjoint paths-a survey, SIAM J. Algebraic Discrete Methods, 6 (1985), pp. 300-305.

15. — Graph minors. V. Excluding a planar graph, J. Comb. Theory Series B, 41 (1986), pp. 92-114.

16. — Graph minors. X. Obstructions to tree-decomposition, J. Combin. Theory Ser. B, 52 (1991), pp. 153-190.

17. - Graph minors. XVI. Excluding a non-planar graph, J. Combin. Theory Ser. B, 89 (2003), pp. 43-76.

18. N. Robertson, P. D. Seymour, and R. Thomas, Quickly excluding a planar graph, J. Combin. Theory Ser. B, 62 (1994), pp. 323-348.

19. C. Thomassen, A simpler proof of the excluded minor theorem for higher surfaces, J. Combin. Theory Ser. B, 70 (1997), pp. 306-311. 